\title{
Editorial: Beyond the Education of Smart Systems Integration
}

\author{
Ferenc Ender ${ }^{1}$ \\ ${ }^{1}$ Guest Editor, Faculty of Electrical Engineering and Informatics, Budapest University of Technology and Economics, \\ H-1521 Budapest, P.O.B. 91, Hungary
}

Smart Systems, such as self-driving cars, artificial pancreas, Internet of Things, smart transportation systems, M2M-enabled advanced manufacturing robots or wellness armbands, combine data processing with multi-modal (optical, biological, mechanical) sensing, actuation and communication. Taking often advantage of miniaturization and/ or combining functionalities and communicate with other "intelligent" systems and devices, smart systems can analyse complex situations to take autonomous decisions or provide real-time data that allow informed operator decision. The integration of Smart Systems into large systems provide key technical advantages for increased products competitiveness in most industrial sectors. However, Europe is the global leader in Smart Systems manufacturing and research, this economic growth and continuous advantages that Smart Systems offer to society can only be sustained by the provision of a pipeline of graduate engineers and scientists.

The Joint International Master in Smart Systems Integration (SSI) is offering an MSc degree covering tomorrow's technologies in Smart Systems with a special focus on how such systems are designed and manufactured through integration of microsystems with microelectronics. The subject is truly inter-disciplinary within engineering, and a single university would typically only be able to cover parts of the relevant topics for such a degree. SSI is given jointly by three institutions: Heriot-Watt University (HWU) (Edinburgh, Scotland), University College of Southeast Norway (HSN), and Budapest University of Technology and Economics (BME) (Hungary), each providing one full taught semester. The SSI program uses the complementary expertise and laboratory facilities of the three institutions to provide a unique program that truly offers a system-level approach to Smart Systems Integration. Being the programme started five years ago, the annual SSI Summer School is organized in Hungary was extended by an opportunity for alumni students to present their research progress some years after graduation. In this issue three papers are presented written by alumni students of the SSI programme who presented their works in the First Symposium of Smart Systems Integration, in August 2018, at Balatonvilágos, Hungary.
The main applications of smart systems technologies are well represented in the issue. Two papers present advanced methods for particle separation in microfluidic devices. This crucial step in smart microdevices enables many industrial, biomedical, clinical or chemical applications. Acoustic methods presented in the papers offer contactless, biocompatible, scalable sorting with high degree of reconfigurability and are therefore favoured techniques. Integration of nano- and microtechnologies is often a key challenge in novel device design. Another paper from the field of biological application of smart systems represents a method to build microfluidic devices with enzyme loaded nanofibers located in the channels. Such devices offer a versatile solution for enzymatic studies or even organ-on-a-chip applications with a favourable and well known highly effective immobilization technique.

Electronics technology and its usage in smart systems integration gives another scope of the current issue. Using polymer substrates for structural electronics opens new possibilities like higher components density, cost effective short series and prototypes or manufacturing of electronics. The paper presented in this issue introduces a novel method to fabricate polymer substrates using 3D printing. Electromagnetic compatibility is remaining a main issue as smart systems mostly communicate with other systems or with each other using wireless technologies. In the current issue we present two papers dealing with EMC issues of RFID-based navigation robots and LED lamps, respectively. The usage of smart systems in transportation is also covered by a paper dealing with the embedded control of an unmanned aerial vehicle.

Smart systems integration effectively links the formerly distinct areas such as electronics technology and control theory or biological systems. The success of education programmes in this field is evident from the large number of applicants, the excellent academic and scientific results the students and graduates obtain, and the high employability rate. A large proportion of SSI alumni students pursues Ph.D. studies in the field of Smart Systems, showing the academic excellence of the graduates. 